LA W RENCE LIVERMORE NATIONAL LABORATORY

\section{Plasma Amplifier contribution for FY09 LDRD Annual Report}

R. Kirkwood

November 19, 2009 
This document was prepared as an account of work sponsored by an agency of the United States government. Neither the United States government nor Lawrence Livermore National Security, LLC, nor any of their employees makes any warranty, expressed or implied, or assumes any legal liability or responsibility for the accuracy, completeness, or usefulness of any information, apparatus, product, or process disclosed, or represents that its use would not infringe privately owned rights. Reference herein to any specific commercial product, process, or service by trade name, trademark, manufacturer, or otherwise does not necessarily constitute or imply its endorsement, recommendation, or favoring by the United States government or Lawrence Livermore National Security, LLC. The views and opinions of authors expressed herein do not necessarily state or reflect those of the United States government or Lawrence Livermore National Security, LLC, and shall not be used for advertising or product endorsement purposes.

This work performed under the auspices of the U.S. Department of Energy by Lawrence Livermore National Laboratory under Contract DE-AC52-07NA27344. 
Proposed Work from last year's proposal

In FY09, we will complete experiments to

demonstrate the compression of a 20- to 30-ps portion of a nanosecond pulse to less than 1-ps duration in a plasma optimized for focal spot quality and efficiency. This will be the first demonstration of pulse compression in such a plasma. These results will build on our FYO7 and FYO8 results in large-scale plasmas with long interaction times. We will also carry out studies of pump beam combination by ion waves with the available resources to facilitate application of plasma pulse compression to large lasers with multiple beams.

Enter accomplishments below:

In FY09 we performed experiments to interact a frequency shifted, short pulse seed developed previously, with 20 ps of the nanosecond Janus pulse and demonstrated amplification factors as high as $60 \mathrm{x}$ at low seed energy. As the seed energy increased the amplification factor was then observed to decrease more rapidly than our pre-experiment 1D pic simulations, limiting the maximum output seed power to $4 \times 10^{\wedge} 9 \mathrm{~W}$, and demonstrating a window in which non-linear effects nearly suppress scattering. Data with 0.5 and $3.5 \mathrm{ps}$ duration seeds confirmed the non-linear effects scaled with power, and is providing a benchmark for on-going 2D PIC simulations (LANL) that will be used to design plasma amplifiers, to take advantage of suppressed scattering to improve coupling in NIC ignition targets.

Summary Statement (required)

Please provide a one-sentence summary statement for your project. For example, "The successful conclusion of this project enabled novel techniques of proton heating and $x$-ray backlighting to create and diagnose high-energy-density solid plasmas".

And

Provide a one-sentence statement of next steps for continuing your research. For example, "The National Institutes of Health will provide support for additional research efforts to generate target and binding site data for transcription factor proteins" or "Our cross-section measurement capability is now being applied to programmatic measurements" or "We have established a CRADA with an industrial partner that 
will fully develop this technology for use in precision machining".

The successful conclusion of this project has provided a data base for designing Raman amplifiers and pulse compressors in hot plasmas and controlling their effects in multi-beam ignition experiments. In particular the data identified high-dimensional nonlinear effects that limit Raman amplification which will both determine amplifier and compressor design in the future and allow for improved coupling in central hot spot ignition targets at NIC.

The NIC program in collaboration with LANL is developing a 2D simulation model from the data obtained in these experiments and will use it to design future experiments which optimize the coupling in hohlraums in the presence of Raman re-amplification due to the multiple intersecting laser beams. 